\title{
Direkte Demokratie in den Ländern - Kontingenter Prozeß oder List der Vernunft?
}

\author{
Fabian Wittreck
}

\section{Einleitung: Der Volksentscheid über „,Stuttgart 21 “ als typologisches Rudi- ment}

Im November 2011 stand im Zentrum der öffentlichen Aufmerksamkeit der Volksentscheid, der den Konflikt um das Bahnhofsprojekt „Stuttgart 21“ beenden sollte ${ }^{1}$ (besondere Beachtung fand allerdings gerade der Streit in der grünroten Koalition, ob das Votum des Volkes in seiner Wirkung nun endgültig, vorläufig oder eben ,,interpretationsbedürftig“ sein solle ${ }^{2}$ ). Nicht allein wegen der singulären Konfliktsituation und der konfligierenden Deutungsmuster dürfte „Stuttgart 21“ ein denkbar schlechtes Anschauungsobjekt für direkte Demokratie in den Ländern sein ${ }^{3}$. Denn die in der Politikwissenschaft einhellig beschriebene

* Überarbeitete und aktualisierte Fassung eines Vortrags, den der Verfasser vor der Deutschen Sektion der Internationalen Juristen-Kommission in Bremen gehalten hat. Mein herzlicher Dank gilt Herrn Priv.-Doz. Dr. Otmar Jung für seine kritischen Anmerkungen sowie den folgenden Mitarbeitern meiner Professur, die sich unter der Anleitung von Herrn Wiss. Mitarbeiter Stephan Wagner um die Verifizierung und Aktualisierung des Fußnotenapparats verdient gemacht haben: Nana K.A. Baidoo, Philipp Cramer, Jan Holze, Benjamin Karras, Jörg Schuirmann und Marcel Wirth.

1 Vgl. dazu nur O. Jung, Stuttgart 21 und die Direkte Demokratie, in: RuP 48 (2012), S. 11 ff.; U. Eith/G. Mielke/D. Oberndörfer, Volksentscheide in der Parteiendemokratie - Das Lehrstück Stuttgart 21, in: Berliner Republik 13/1 (2012), S. 60 ff.; F. Decker, Möglichkeiten und Grenzen der direkten Demokratie: das Beispiel Stuttgart 21, in: S. Braun (Hrsg.), Die verstimmte Demokratie. Moderne Volksherrschaft zwischen Aufbruch und Frustration, 2012, S. 209 ff.; M. Fatke/M. Freitag, Die direkte Demokratie in BadenWürttemberg und Stuttgart 21, in: Der Bürger im Staat 62 (2012), S. 174 ff.; C. Franzius, Stuttgart 21: Eine Epochenwende?, in: GewArch. 2012, S. 225 ff.; V.M. Haug, Volksgesetzgebung auf verfassungsrechtlichem Neuland, in: ZParl. 43 (2012), S. 446 ff.; zuletzt A. Schäfer/C. Adam/M. Schlichenmaier, Das Plebiszit über das Bahnhofsprojekt „Stuttgart 21“, in: ZPol 22 (2012), S. 187 ff. - Aus der Literatur im Vorfeld W. Leisner, „Wir sind das Volk!“ - Wer?, in: NJW 2011, S. 33 ff.; G. Hermes/J. Wieland, Rechtliche Möglichkeiten des Landes Baden-Württemberg, die aus dem Finanzierungsvertrag „Stuttgart 21 “ folgenden Verpflichtungen durch Kündigung oder gesetzliche Aufhebung auf der Grundlage eines Volksentscheides zu beseitigen, in: L.P. Feld/P.M. Huber/O. Jung/C. Welzel/F. Wittreck (Hrsg.), Jahrbuch für direkte Demokratie 2010, 2011, S. 350 (380 ff.). Siehe nur B. Wieselmann, Stuttgart-21-Gegner wollen Quorum nicht akzeptieren, in: Badische Zeitung v. 4.11.2011.

3 Statt aller Decker, Möglichkeiten (Fn. 1), S. 213 f. 
konsensbildende Vorwirkung plebiszitärer Instrumente ${ }^{4}$ konnte der Volksentscheid angesichts längst verhärteter Fronten nicht mehr entfalten. Und in verfassungsgeschichtlicher Perspektive weist die Idee, das Volk als Schiedsrichter im „Streit“ zwischen dem Parlament und der von diesem mehrheitlich getragenen Regierung in die Schranken zu rufen, tief in das 19. Jahrhundert zurück ${ }^{5}$. Von der aberwitzigen Volte schließlich, im quasi laufenden Verfahren noch rasch das Quorum auf just das Niveau zu senken, das man mit der eigenen Anhängerschaft glaubte erreichen zu können ${ }^{6}$, darf man wohl nur betreten schweigen.

Im folgenden wird demgegenüber zunächst zu belegen sein, daß die derzeit noch geltende baden-württembergische Regelung hinter dem Stand der direkten Demokratie in den übrigen Ländern markant zurückbleibt (II.). Insbesondere ist zu zeigen, daß der Südweststaat bislang praktisch keinen Anteil an einer unübersehbaren Entwicklung hatte, die bereits als „Siegeszug“ der direkten Demokratie apostrophiert worden ist ${ }^{7}$ (III.). Nach der Benennung einzelner retardierender Faktoren bzw. rechtspolitischer „Baustellen“ (IV.) wird deshalb abschließend zu fragen sein, ob sich derzeit ein (deutsches?) Idealmodell direkter Demokratie ab-

Siehe nur $O$. Jung, Wenn der Souverän sich räuspert ... Vorwirkungen direktdemokratischer Korrekturmöglichkeiten, dargestellt an Beispielen aus Nordrhein-Westfalen, Niedersachsen und Rheinland-Pfalz, in: Jahrbuch zur Staats- und Verwaltungswissenschaft 8 (1995), S. 107 (108 ff., 157 ff.); A. Christmann, Damoklesschwert Referendum? Die indirekte Wirkung ausgebauter Volksrechte auf die Rechte religiöser Minderheiten, in: Schweizerische Zeitschrift für Politikwissenschaft 16 (2010), S. 1 (12). - Instruktiv zuletzt L. Bernhard/H. Kriesi, Coalition Formation, in: H. Kriesi (Hrsg.), Political Communication in Direct Democratic Campaigns: Enlightening or Manipulating?, Basingstoke u.a. 2012 , S. $54 \mathrm{ff}$.

5 Die dem Volksentscheid zugrundeliegende Bestimmung des Art. 60 Abs. 2 BWVerf. perpetuiert in der Sache Vorstellungen des Konstitutionalismus, da sie von einer Frontstellung zwischen der Volksvertretung und der (monarchischen) Exekutive ausgeht, die dem parlamentarischen Regierungssystem in dieser Form fremd ist bzw. allenfalls im Falle einer Minderheitsregierung ausnahmsweise Sinn ergeben mag. Zugleich tritt das Volk hier als funktionales Äquivalent zu der dem Konstitutionalismus noch fremden Verfassungsgerichtsbarkeit auf; näher unten II.2. - Instruktiv zum zeitgenössischen Verfassungsverständnis E.-W. Böckenförde, Der Verfassungstyp der deutschen konstitutionellen Monarchie im 19. Jahrhundert, ders. (Hrsg.), Moderne deutsche Verfassungsgeschichte, 1972, S. $146 \mathrm{ff}$.

6 Vgl. den Gesetzentwurf der Grünen zur Änderung der Landesverfassung vom 7.7.2011 (LT-Drs. 15/216) mit dem Ziel, das Zustimmungsquorum von einem Drittel (so noch Art. 60 Abs. 5 S. 2 LVerf.) auf ein Viertel zu senken; dazu nur S. Fischer/S. Weiland, Streit um S21-Volksentscheid: Grüne fürchten das Volk, in: Spiegel-Online v. 21.4.2011.

Diese Wendung bei $O$. Jung, Siegeszug direktdemokratischer Institutionen als Ergänzung des repräsentativen Systems? Erfahrungen der 90er Jahre, in: H.H. v. Arnim (Hrsg.), Demokratie vor neuen Herausforderungen, 1999, S. 103 (103) sowie R. Geitmann, Der Siegeszug der kommunalen Direktdemokratie, in: H.K. Heußner/O. Jung (Hrsg.), Mehr direkte Demokratie wagen, 1. Aufl. 1999, S. 237 (237). 
zeichnet, dem die Landesverfassungen mit einer gewissen historischen Notwendigkeit zustreben (V.).

\section{Direkte Demokratie in den Ländern: Bestandsaufnahme}

Fragen wir zunächst nach dem status quo. Er ist gekennzeichnet durch eine mittlerweile flächendeckende Einführung der Volksgesetzgebung in den Ländern (1.). Deutlich seltener findet sich die Möglichkeit der nachträglichen Einflußnahme auf die Volksvertretung in Gestalt der Parlamentsauflösung oder des eingangs skizzierten Stichentscheids (2.). Nur als Ausnahme begegnet das Referendum, in der Regel dann als obligatorische Abstimmung über Verfassungsänderungen (3.). Außer Betracht bleiben im folgenden die kommunale Ebene, also Bürgerbegehren und Bürgerentscheid ${ }^{8}$, die intensiv diskutierte Einfügung direktdemokratischer Elemente in das Grundgesetz ${ }^{9}$ sowie die Frage nach der grundsätzlichen Zulässigkeit direkter Demokratie auf Landesebene - sie dürfte nur noch Gegenstand der neueren Wissenschaftsgeschichte der Disziplin des Öffentlichen Rechts $\operatorname{sein}^{10}$.

8 Dazu materialreich P. Neumann, Sachunmittelbare Demokratie im Bundes- und Landesverfassungsrecht unter besonderer Berücksichtigung der neuen Länder, 2009, S. 117 ff.; vgl. ferner F.-L. Knemeyer, Bürgerbeteiligung und Kommunalpolitik, 2. Aufl. 1997, S. 103 ff.; V. Mittendorf, Bürgerbegehren und Bürgerentscheide in Deutschland, in: H.K. Heußner/O. Jung (Hrsg.), Mehr direkte Demokratie wagen, 2. Aufl. 2009, S. 327 ff.; $C$. Eder, Direkte Demokratie in den deutschen Kommunen: in: M. Freitag/A. Vatter (Hrsg.), Vergleichende subnationale Analysen für Deutschland, 2010, S. 65 ff.; B. Aker, Instrumente direkter Demokratie - Bürgerbegehren und Bürgerentscheid, in: VBIBW 2011, S. 455 ff. - Statistische Angaben bei T. Schiller, Direkte Demokratie auf Bundesländer- und Kommunalebene, in: M. Freitag/U. Wagschal (Hrsg.), Direkte Demokratie, 2007, S. 115 (128 ff.). - Siehe zuletzt - wenn auch streckenweise einseitig - H.-G. Henneke, Wutbürger in Verantwortung, in: DVB1. 2012, S. 1072 (1075 ff.).

9 Dazu nur einige neuere Stellungnahmen: Restriktiv argumentieren P. Badura, Plebiszitäre Ergänzung oder Verformung des parlamentarischen Regierungssystems in der Bundesverfassung, in: M. Ruffert (Hrsg.), Dynamik und Nachhaltigkeit des Öffentlichen Rechts. Festschrift für Meinhard Schröder, 2012, S. 307 ff. sowie S. Müller-Franken, Plebiszitäre Gesetzgebung in das Grundgesetz?, in: P. Neumann/D. Renger (Hrsg.), Sachunmittelbare Demokratie im interdisziplinären und internationalen Kontext 2009/2010, 2012, S. 48 (51 ff.); offenes Votum für die (einfachgesetzliche) Einführung von Volksbegehren und Volksentscheid hingegen bei H. Meyer, Volksabstimmungen im Bund: Verfassungslage nach Zeitgeist?, in: JZ 2012, S. 538 ff.

10 Klassisch die Studie von H. Hofmann, Bundesstaatliche Spaltung des Demokratiebegriffs?, in: W. Barfuß u.a. (Hrsg.), Festschrift für Karl H. Neumayer, 1985, S. 281 ff.; zuletzt pointiert für die Zulässigkeit direkter Demokratie M. Paus/A. Schmidt, Das Grundgesetz und die direkte Demokratie auf staatlicher und kommunaler Ebene, in: JA 2012, S. 48 (49 f.). - Die These, daß direkte Demokratie in den Ländern verfassungswidrig sei, vertritt ernstlich niemand mehr; erhebliche verfassungspolitische Bedenken äußern nach 
Archetypus direkter Demokratie in den Ländern ist die Gesetzgebung durch das Volk im Wege von Volksbegehren und Volksentscheid; sie begegnet inzwischen lückenlos ${ }^{11}$. Teils erhebliche Unterschiede weisen die Hürden (dazu sogleich unter III.), die Vorabkontrolle ${ }^{12}$ durch Landtag, Exekutive oder Gerichte sowie die jeweiligen Ausschlußtatbestände auf ${ }^{13}$.

Diese Fixierung auf die als „Gaspedal“ apostrophierte Gesetzgebung ist unlängst von politikwissenschaftlicher Seite kritisiert worden; aus der Beobachtung, daß Volksgesetzgebung bislang überwiegend Vetopositionen besetzt hat, schlußfolgert Frank Decker, daß eine als „Bremse“ fungierende Referendumslösung besser mit dem real existierenden Repräsentativsystem zu verzahnen wä$\mathrm{re}^{14}$. Derartige Gesetzesreferenden (verstanden als vergleichsweise niedrigquori-

wie vor $P$. Krause, Verfassungsrechtliche Möglichkeiten unmittelbarer Demokratie, in: J. Isensee/P. Kirchhof (Hrsg.), Handbuch des Staatsrechts der Bundesrepublik Deutschland, Bd. III, 3. Aufl. 2005, §35 Rn. 17 ff.; J. Isensee, Demokratie ohne Volksabstimmung: das Grundgesetz, in: C. Hillgruber/C. Waldhoff (Hrsg.), 60 Jahre Bonner Grundgesetz eine geglückte Verfassung?, 2010, S. 117 (120 ff.) sowie S. Müller-Franken, Die Geister, die ich rief. Zu Risiken und Nebenwirkungen von Plebisziten, in: H. Meier/G. Panzer (Hrsg.), Direkte Demokratie im Grundgesetz?, 2010, S. 45 (50 ff. u. passim). - Kondensat der Diskussion bis zur Jahrtausendwende bei C. Schwieger, Volksgesetzgebung in Deutschland, 2005, S. $351 \mathrm{ff}$.

11 Zusammenfassende Darstellungen bei J. Rux, Direkte Demokratie in Deutschland, 2008, S. 265 ff., 414 ff.; G. Jürgens/F. Rehmet, Direkte Demokratie in den Bundesländern Ein Überblick, in: Heußner/Jung, Demokratie² (Fn. 8), S. 197 ff.; C. Eder, Direkte Demokratie auf subnationaler Ebene, 2010, S. 85 ff.; F. Decker, Zwischen Placebo und Erfolgsmodell, in: ZParl. 41 (2010), S. 564 ff.; A. Rohner, Direct Democracy in the German Länder: History, Institutions, and (Mal)Functions, 2011, abrufbar unter: http://www.c2d.ch/files/C2D_WP38.pdf (27.12.2012); H. Schmitz, Volksgesetzgebung, in: DVBl. 2012, S. 731 (732 ff.). sowie jüngst H. Obermann, Entwicklung direkter Demokratie im Ländervergleich, in: LKV 2012, S. 241 ff.

12 Siehe dazu S. Przygode, Die deutsche Rechtsprechung zur unmittelbaren Demokratie, 1995, S. 73 ff.; A. Stiens, Chancen und Grenzen der Landesverfassungen im deutschen Bundesstaat der Gegenwart, 1997, S. 215 ff. sowie Rux, Demokratie (Fn. 11), S. 292 ff., 418 ff. - Näher unten IV.3.

13 Im Überblick R. Magin/C. Eder, Direkte Demokratie in den Bundesländern, in: Freitag/Wagschal, Demokratie (Fn. 8), S. 151 (167 ff.); vgl. ferner Rux, Demokratie (Fn. 11), S. $271 \mathrm{ff}$., $414 \mathrm{f}$.

14 F. Decker, Volksgesetzgebung oder Volksveto?, in: Feld u.a., Jahrbuch 2010 (Fn. 1), S. 37 ff.; Gegenposition bei W.J. Patzelt, Welche plebiszitären Instrumente können wir brauchen?, ebda., S. 63 ff. (insb. S. 75 f., 98 ff.) sowie F. Wittreck, Volksgesetzgebung: Königsweg oder Irrweg der direkten Demokratie?, in: ZSE 8 (2010), S. 553 (559 f.). Zusammenfassend S. Müller-Franken, Referendum versus Volksgesetzgebung, in: R.T. Baus (Hrsg.), Perspektiven und Grenzen „,direkter Demokratie“, 2012, S. 51 ff., der selbst der Einführung obligatorischer Referenden zu ausgewählten Themen zuneigt (ebda., S. $60)$. 
ge Möglichkeit des Volkes, ein parlamentsbeschlossenes Gesetz lediglich aufzuheben $^{15}$ ) sind bislang die Ausnahme. Noch dazu hängen sie von weiteren Voraussetzungen ab (in Hamburg der Änderung eines volksbeschlossenen Gesetzes $^{16}$, in Baden-Württemberg dem Antrag einer Parlamentsminderheit sowie dem Beschluß der Regierung ${ }^{17}$, in Bremen dem Mehrheitsbeschluß der Bürgerschaft ${ }^{18}$ und in Rheinland-Pfalz von einem Aussetzungsverlangen der Parlamentsminderheit sowie zusätzlich einem Volksbegehren ${ }^{19}$ ) bzw. stellen sich als funktionale Äquivalente zur Verfassungsgerichtsbarkeit dar (sogleich unter 2.). Ob eine generelle Ergänzung des direktdemokratischen Instrumentariums um ein allgemeines Referendum rechtspolitisch sinnvoll sein mag, bleibe hier dahingestellt; das Volk umgekehrt de constitutione ferenda auf solche „Vetoinitiativen“ zu beschränken, dürfte sich zumindest als verfassungspädagogisch fragwürdig erwei$\operatorname{sen}^{20}$.

15 Sie begegnen prominent in der Schweiz (vgl. auf Bundesebene Art. 140 f. BV): H.K. Heußner/O. Jung, Die direkte Demokratie in der Schweiz, in: dies., Demokratie ${ }^{2}$ (Fn. 8), S. 115 (122 f.); A. Tschentscher, Direkte Demokratie in der Schweiz - Länderbericht 2008/2009, in: L.P. Feld/P.M. Huber/O. Jung/C. Welzel/F. Wittreck (Hrsg.), Jahrbuch für direkte Demokratie 2009, 2010, S. 205 (205 f., 207 f., 209 ff., 228 f.); jüngster Überblick zu hängigen Verfahren bei $A$. Tschentscher/D. Blonski, Direkte Demokratie in der Schweiz - Länderbericht 2010/2011, in: L.P. Feld/P.M. Huber/O. Jung/H.-J. Lauth/F. Wittreck (Hrsg.), Jahrbuch für direkte Demokratie 2011, 2012, S. 139 (152 ff.: auf Bundesebene ein Referendum [S. 160] gegenüber einer Vielzahl von Initiativen).

16 Vgl. Art. 50 Abs. 4 HambVerf. und dazu A. v. Arnauld, „Refolution“ an der Elbe: Hamburgs neue direkte Demokratie, in: Feld u.a., Jahrbuch 2009 (Fn. 15), S. 90 (117 f.); $O$. Jung, Volksgesetze und parlamentarische Konterlegislatur, in: K.H. Schrenk/M. Soldner (Hrsg.), Analyse demokratischer Regierungssysteme. Festschrift für Wolfgang Ismayr, 2010, S. 427 (434 ff.) sowie A. Dressel, Direkte Demokratie in Hamburg, in: V. Mehde/U. Ramsauer/M. Seckelmann (Hrsg.), Staat, Verwaltung, Information. Festschrift für Hans Peter Bull, 2011, S. 107 (109 f.). - Näher zum Problem unten unter IV.1.

17 Vgl. Art. 60 Abs. 2 und 3 BWVerf. und dazu K. Braun, Kommentar zur Verfassung des Landes Baden-Württemberg, 1984, Art. 60 Rn. 13 ff. sowie P. Feuchte, in: ders. (Hrsg.), Verfassung des Landes Baden-Württemberg, 1987, Art. 60 Rn. 4 ff.

18 Vgl. Art. 70 Abs. 1 lit. a und b BremVerf. (lit. a betrifft Verfassungsänderungen [Mehrheit der Mitglieder], lit. b andere der Zuständigkeit der Bürgerschaft unterliegende Fragestellungen [einfache Mehrheit]) und dazu kritisch D. Schefold, Die Neuregelung des Volksentscheids in Bremen vom 1. September 2009, in: Feld u.a., Jahrbuch 2010 (Fn. 1), S. 135 (141: ,... nichts spricht dafür, dass diese Regelung in Zukunft praktisch relevanter wird als in den letzten sechzig Jahren“").

19 Siehe Art. 114 S. 1 und Art. 115 Abs. 1 RPVerf. und dazu D. Franke, in: C. Grimm/P. Caesar (Hrsg.), Verfassung für Rheinland-Pfalz, 2001, Art. 114 Rn. 8 f. sowie zuvor Jung, Souverän (Fn. 4), S. 146 f., 172 f.: Konkret gelangt ein Gesetz dann zur Abstimmung, wenn ein Drittel der Landtagsmitglieder seine Aussetzung verlangt und mindestens 150.000 Stimmberechtigte in einem Volksbegehren für das Referendum votieren.

20 Wie hier und ausgiebig zu den Gründen Patzelt, Instrumente (Fn. 14), S. 98 ff. 
Eindeutig auf dem Vormarsch ist zuletzt die Volksinitiative (auch Bürgerantrag o.ä. genannt ${ }^{21}$ ), verstanden als Antrag, das Parlament mit einer Frage der politischen Willensbildung zu befassen; sie steht teils neben der eigentlichen Volksgesetzgebung $^{22}$, kann teils in dieses Verfahren übergeleitet werden ${ }^{23}$. Derzeit kennen zwölf Landesverfassungen vergleichbare Verfahren, mehrheitlich des ersten Typs ${ }^{24}$.

\section{Parlamentsauflösung und Stichentscheid}

Dritthäufigste direktdemokratische Option ist (wohlgemerkt dem Normtext nach) die Auflösung des Parlaments ${ }^{25}$ bzw. die funktional gleichwertige Abkürzung der Wahlperiode $^{26}$. Sie ist typischer Bestandteil der vorgrundgesetzlichen Landesverfassungen, während die neuen Bundesländer hier deutliche Zurückhaltung zeigen bzw. allenfalls zur Abgeordnetenanklage Zuflucht nehmen ${ }^{27}$. Derzeit könnten die Bürger in sechs Ländern (Baden-Württemberg, Bayern, Berlin, Brandenburg,

21 Eine derartige abweichende Terminologie in Bremen (,Anträge auf Beratung und Beschlußfassung"; Art. 87 Abs. 1 LVerf.), Hamburg („Volkspetition“, Art. 29 LVerf.), Sachsen („Volksantrag, Art. 71 LVerf.) sowie in Thüringen („Bürgerantrag“; Art. 68 LVerf.). - In Berlin („Einwohnerinitiative“, Art. 61 LVerf.) geht die Abweichung über den Wortlaut hinaus, weil hier auf der ersten Stufe die Einleitungsberechtigung vom Bürgerstatus entkoppelt wird.

22 So in Berlin (Art. 61 LVerf.), Bremen (Art. 87 LVerf.), Mecklenburg-Vorpommern (Art. 59 LVerf.), Niedersachsen (Art. 47 LVerf.), Nordrhein-Westfalen (Art. 67a LVerf.), Rheinland-Pfalz (Art. 108a LVerf.), Sachsen-Anhalt (Art. 80 LVerf.) und Thüringen (Art. 68 LVerf.); vgl. jeweils Rux, Demokratie (Fn. 11), S. 598 ff., 633 ff., 664 ff., 697 ff., 724 ff., 765 ff., 802 ff., 853 ff., 873 ff.

23 Diese Handhabung in Brandenburg (Art. 76, 77 Abs. 1 LVerf.), Hamburg (Art. 50 Abs. 2 LVerf.), Sachsen (Art. 71, 72 Abs. 1 LVerf.) und Schleswig-Holstein (Art. 41, 42 Abs. 1 LVerf.).

24 Zusammenfassend Rux, Demokratie (Fn. 11), S. $406 \mathrm{ff}$.

25 Vgl. Art. 43 Abs. 2 BWVerf., Art. 18 Abs. 3 BayVerf., Art. 76 Abs. 1 S. 2, 77 Abs. 3 S. 2, 78 Abs. 3 S. 1 BbgVerf. sowie Art. 109 Abs. 1 Nr. 2 RPVerf.; zusammenfassend wiederum Rux, Demokratie (Fn. 11), S. 503 ff. sowie Rohner, Democracy (Fn. 11).

26 Siehe 70 Abs. 1 lit c, 76 Abs. 1 lit. b, 2 BremVerf. sowie Art. 62 Abs. 3, 63 Abs. 3 BerlVerf.

27 Sie begegnet in Brandenburg (Art. 61 LVerf.) und Sachsen (Art. 118 LVerf.); vgl. als erstes Anschauungsmaterial SächsVerfGH, Beschl. v. 11.12.2008, Az. Vf. 151-IX-07, DÖV 2009, 210 und dazu B. Kunzmann, Die Sächsische Verfassung in der Übergangszeit oder: zeit- und unzeitgemäße Gedanken zu Art. 118 f. SächsVerf, in: SächsVBl. 2009, S. 105 f. Siehe ferner Art. 42 BWVerf., Art. 61 Abs. 1 u. 3 BayVerf., Art. 17 NdsVerf. sowie Art. 85 SaarlVerf. - Eine zusammenfassende Darstellung fehlt; vgl. allerdings Sobolewski, Das Instrument der Abgeordnetenanklage in den Ländern der Bundesrepublik, 1992 (Wiss. Dienste des Deutschen Bundestages, Ausarbeitung Nr. 174/92). 
Bremen und Rheinland-Pfalz) auf diese Weise direkt Zugriff auf ihre Repräsentanten nehmen ${ }^{28}$.

Besonderer Aufmerksamkeit bedürfen in diesem Kontext die Regelungen in Baden-Württemberg (eingangs bereits angesprochen) ${ }^{29}$ und NordrheinWestfale $^{30}$. Hier kann das Volk zum Schiedsrichter in einem (echten oder fingierten) Streit zwischen Landtag und Regierung berufen werden. Die Folgen sind allerdings zumindest in Nordrhein-Westfalen gravierend. Denn das Volk entscheidet nicht nur über das Zustandekommen des zwischen den Kontrahenten umstrittenen Gesetzes, sondern auch über deren weiteres Schicksal: Obsiegt die Landesregierung, kann sie den Landtag auflösen; unterliegt sie, muß sie zurück$\operatorname{treten}^{31}$. Ob dieses der Funktionslogik des Konstitutionalismus verhaftete ${ }^{32}$ (und

28 Ein Blick in die Verfassungspraxis belegt zugleich, daß das Instrument weitgehend inert geblieben ist; konkret haben in Berlin 1981 und 2001 bereits erste Unterschriftensammlungen zur Selbstauflösung des Abgeordnetenhauses geführt: O. Jung, Direkte Demokratie in Berlin. Der Fall „Pro Reli“ 2007-2009, 2011, S. 40 f.; ders., Die Reform der direkten Demokratie in Berlin 2006 (Teil I), in: Feld u.a., Jahrbuch 2011 (Fn. 15), S. 259 (261). - In Baden-Württemberg verfehlten die Befürworter beim bislang bundesweit einzigen Auflösungsentscheid 1971 das Zustimmungsquorum: O. Jung, Daten zu Volksentscheiden in Deutschland auf Landesebene (1946-1992), in: ZParl. 24 (1993), S. 5 (11); vgl. noch H.-G. Wehling, Direkte Demokratie in Baden-Württemberg, in: A. Kost (Hrsg.), Direkte Demokratie in den deutschen Ländern. Eine Einführung, 2005, S. 14 (24).

29 Siehe Art. 60 Abs. 2 und 3 BWVerf. und dazu außer der in Fn. 17 zitierten Literatur noch Wehling, Demokratie (Fn. 28), S. 15. - Zur Entstehung knapp P. Feuchte, Verfassungsgeschichte von Baden-Württemberg, 1983, S. 217.

30 Siehe Art. 68 Abs. 3 NWVerf. und speziell dazu D. Grimm, Verfassungsrecht, in: ders./H.-J. Papier (Hrsg.), Nordrhein-westfälisches Staats- und Verwaltungsrecht, 1986, S. 1 (43); T. Mann, in: W. Löwer/P.J. Tettinger (Hrsg.), Kommentar zur Verfassung des Landes Nordrhein-Westfalen, 2002, Art. 68 Rn. 46 ff.; A. Kost, Direkte Demokratie in Nordrhein-Westfalen, in: ders., Demokratie (Fn. 28), S. 183 (183); C. Günther, in: A. Heusch/K. Schönenbroicher (Hrsg.), Die Landesverfassung Nordrhein-Westfalen, 2010, Art. 68 Rn. 19 ff.; R. Grawert, Verfassung für das Land Nordrhein-Westfalen, 3. Aufl. 2012, Art. 68 Anm. 3. - Zur Entstehungsgeschichte instruktiv W. Kringe, Machtfragen, 1988, S. $500 \mathrm{ff}$.

31 Kritisch A. Kost, Direkte Demokratie an Rhein und Ruhr, in: Heußner/Jung, Demokratie 2 (Fn. 8), S. 257 (270) sowie J. Dietlein, Verfassungsrecht, in: ders./M. Burgi/J. Hellermann, Öffentliches Recht in Nordrhein-Westfalen, 4. Aufl. 2011, § 1 Rn. 168: ,kaum geeignet".

32 Vgl. oben Fn. 5. Die Stichentscheide teilen diese Schwäche mit der Ministeranklage, die ebenfalls ihren „Sitz im Leben“ im konstitutionalistischen Denken hat, eingedenk der Möglichkeit des Mißtrauensvotums aber inzwischen schlicht redundant ist. Vgl. hierzu jüngst eingehend S. Steinbarth, Das Institut der Präsidenten- und Ministeranklage in rechtshistorischer und rechtsvergleichender Perspektive, 2011, S. 72 ff.; für eingeschränkt sinnhaft hält das Instrument auch L. Freund, Die Anklageverfahren vor den Landesverfassungsgerichten, in: C. Starck/K. Stern (Hrsg.), Landesverfassungsgerichtsbarkeit, Bd. II, 1983, S. 307 (345). 
in der Zwischenkriegszeit noch vergleichsweise häufige ${ }^{33}$ ) Modell heute wenigstens für die lange Zeit ja unvorstellbaren Minderheitsregierungskonstellationen noch einen Restsinn entfaltet ${ }^{34}$, mag man diskutieren.

\section{Verfassungsreferendum}

Es bleibt die obligatorische Abstimmung über die Verfassung als Ganzes oder deren spätere Änderungen ${ }^{35}$. Im Prozeß der Verfassunggebung hat das Volk an neun geltenden deutschen Landesverfassungen direkt mitgewirkt ${ }^{36}$; zur verpflichtenden Abstimmung über Eingriffe in den Text ist es nur in Bayern und Hessen (hier demnächst zu gleichwertigen Lebensverhältnissen und vier weiteren Punkten ${ }^{37}$, dort zuletzt im Frühjahr 2011 zur Schuldenbremse ${ }^{38}$ ) berufen. In

33 Texte in: F. Wittreck (Hrsg.), Weimarer Landesverfassungen, 2004; zusammenfassend mit Einzelnachweisen ders., Zur Einleitung: Verfassungsentwicklung zwischen Novemberrevolution und Gleichschaltung, ebda, S. 1 (16 f., 49 f.) sowie Rux, Demokratie (Fn. 11), S. $161 \mathrm{ff}$.

34 Dieser Hinweis bei Rux, Demokratie (Fn. 11), S. 402.

35 Grundlegend zum Verfassungsreferendum K. Loewenstein, Verfassungslehre, 1959, S. 139. Vgl. ferner $O$. Jung, Regieren mit dem obligatorischen Verfassungsreferendum: Wirkung, Konterstrategie, Nutzungsversuche und Umgangsweise, in: ZParl. 36 (2005), S. 161 ff.; S. Tierney, Constitutional referendums, Oxford 2012; L. Morel, Referendum, in: M. Rosenfeld/A. Sajó (Hrsg.), The Oxford Handbook of Comparative Constitutional Law, Oxford 2012, S. 501 (508 f., 510 ff.) sowie zuletzt M. Böckenförde, Die Einbindung der Bevölkerung in Verfassungsänderungsprozessen - ein Überblick, in: H.P. Hestermeyer u.a. (Hrsg.), Coexistence, Cooperation and Solidarity. Liber Amicorum Rüdiger Wolfrum, Bd. 2, Leiden 2012, S. 1107 ff.

36 Dies sind im einzelnen Bayern (Volksabstimmung 1946), Berlin (1995; vgl. Art. 101 Abs. 1 S. 1 LVerf.), Brandenburg (1992; vgl. den Vorspruch der LVerf.), Bremen (1947; vgl. Art. 155 Abs. 1 LVerf.), Hessen (1946; vgl. Art. 160 Abs. 1 S. 1 LVerf.), Mecklenburg-Vorpommern (1994), Nordrhein-Westfalen (1950; vgl. Art. 90 LVerf.), RheinlandPfalz (1947; vgl. Art. 144 Abs. 1 LVerf.) sowie Thüringen (1994; vgl. Art. 106 Abs. 1 S. 2 LVerf.); zusammenfassend F.R. Pfetsch, Ursprünge der zweiten Republik, 1990, S. 56 ff. sowie O. Jung, Die Landesverfassungsreferenden des Jahres 1994. Daten und Probleme, in: LKV 1995, S. 319 ff.

37 Siehe den parteiübergreifenden Entwurf eines Gesetzes zur Änderung der Verfassung des Freistaates Bayern v. 10.12.2012, LT-Drs. 16/15140, der folgende Neuerungen mit sich bringt: Förderung gleichwertiger Lebensverhältnisse und Arbeitsbedingungen (Art. 3 n.F.); Stärkung der Mitwirkungsrechte des Landtags in Angelegenheiten der Europäischen Union (Art. 70 n.F.); Schuldenbremse (Art. 82 n.F.); angemessene Finanzausstattung der Gemeinden (Art. 83 n.F.); Förderung des ehrenamtlichen Einsatzes für das Gemeinwohl (Art. 121 n.F.).

Siehe Art. 1 des Änderungsgesetzes v. 29.4.2011 (GVB1. I S. 182) und dazu HessStGH, Urt. v. 9.3.2011 - Az. P. St. 2320, NVwZ 2011, 811 sowie - kritisch - K. Eicker-Wolf, Schuldenbremse mit Verfassungsrang? Die politischen Motive der Volksabstimmung in Hessen, in: Forum Wissenschaft 27 (2011), S. 51 ff. 
Bremen kann die Bürgerschaft dem Volk Verfassungsänderungen zur Abstimmung unterbreiten, wenn im Hause keine Zweidrittelmehrheit zustande kommt ${ }^{39}$; Nordrhein-Westfalen räumt die gleiche Option Landtag und Regierung ein ${ }^{40}$. In Baden-Württemberg und Sachsen besteht ebenfalls die Möglichkeit des „von oben“ angeordneten Verfassungsreferendums ${ }^{41}$. In Berlin schließlich muß das Volk lediglich befragt werden, wenn die Verfassungsregeln über Volksbegehren und Volksentscheid selbst geändert werden sollen ${ }^{42}$.

Der Effekt des obligatorischen Verfassungsänderungsreferendums ist evi$\operatorname{dent}^{43}$ : Von der nachgerade triebhaften Änderungsfrequenz des Grundgesetzes ${ }^{44}$ heben sich die beiden Landesverfassungen von 1946 wohltuend ab. Ob die von namhaften Kollegen beklagte „Versteinerung“ der hessischen Verfassung ${ }^{45}$ man denkt hier unwillkürlich an die affirmative Erwähnung der Todesstrafe ${ }^{46}$ dem obligatorischen Referendum oder eher Atavismen einer distinkt konfrontativen Parteienlandschaft ${ }^{47}$ geschuldet ist, sei hier dahingestellt.

39 Siehe Art. 70 Abs. 1 lit. a, Art. 125 Abs. 3 BremVerf und dazu Schefold, Neuregelung (Fn. 18), S. 135 ff., 141.

40 Art. 69 Abs. 3 S. 1 NWVerf. und dazu T. Mann, in: Löwer/Tettinger, Kommentar (Fn. 30), Art. 69 Rn. 12 ff.; C. Günther, in: Heusch/Schönenbroicher, Landesverfassung (Fn. 30), Art. 69 Rn. 15.

41 Siehe Art. 64 Abs. 3 S. 1 BWVerf. bzw. Art. 74 Abs. 3 S. 1 SächsVerf. Beide Bestimmungen sehen einen Antrag von mehr als der Hälfte der Mitglieder des Landtags vor; in der Sache stellen sie damit wie die Regelungen in Bremen und Nordrhein-Westfalen auf das fehlende Zustandekommen der an sich notwendigen Zweidrittelmehrheit ab. Anders P. Feuchte, in: ders., Verfassung (Fn. 17), Art. 64 Rn. 13, der den Sinn der Vorschrift in der - an sich unnötigen - Bestätigung einer im Konsens gefundenen Verfassungsänderung durch das Volk erblickt.

42 Vgl. Art. 100 S. 2 BerlVerf. und dazu H. Lemmer, in: G. Pfennig/M.J. Neumann (Hrsg.), Verfassung von Berlin, 3. Aufl. 2000, Art. 100 Rn. 4; H.-J. Driehaus, in: ders. (Hrsg.), Verfassung von Berlin, 3. Aufl. 2009, Art. 100 Rn. 4; C. Pestalozza, Auf gutem Weg: Direkte Demokratie in Berlin, in: Feld u.a., Jahrbuch 2009 (Fn. 15), S. 295 (313 ff.) sowie Jung, Reform (Fn. 28), S. 281 ff.

43 Kritisch allerdings Jung, Verfassungsreferendum (Fn. 35), S. 167 ff., der eine „stabilisierende Wirkung“ obligatorischer Verfassungsänderungsreferenden zwar für plausibel, aber nicht empirisch belegbar hält und auf die (Mit-)Ursächlichkeit anderer Faktoren wie jeweils unterschiedliche politische Kulturen oder Reformbedürftigkeit hinweist.

44 Siehe F. Wittreck, Die Entwicklung des Grundgesetzes seit 1949, in: Ad Legendum 2011, S. 1 (2 f.).

45 So Michael Stolleis in einem Brief an den Verf. vom 19. März 2001.

46 Dazu, daß weder Art. 21 Abs. 1 S. 2 HessVerf. (Vorbehalt der Todesstrafe für besonders schwere Verbrechen) noch Art. 109 Abs. 1 S. 3 HessVerf. (Bestätigung eines Todesurteils durch die gesamte Landesregierung) gegen Art. $102 \mathrm{GG}$ verstoßen, näher $F$. Wittreck, Die Todesstrafe in den deutschen Landesverfassungen, in: JöR 49 (2001), S. 157 (207 ff., 212 ff.).

47 In diese Richtung H. Meyer, Warum brauchen wir und wie kommen wir zu einer modernen Verfassung?, in: KritV 79 (1996), S. 145 (151); vgl. zur traditionell konfrontativen politischen Situation in Hessen R. Schmitt-Beck/T. Faas, Die hessische Landtagswahl 
In einem nächsten Schritt gilt es zu fragen, ob dieser status quo womöglich gar nicht statisch ist, sondern sich Bewegungsmuster beobachten lassen. Drei scheinen vergleichsweise deutlich aufzuscheinen: Zunächst erleben wir einen Ausbau oder besser eine Ausdifferenzierung der Instrumente der direkten Demokratie (1.). Zweitens sind nahezu flächendeckend die Hürden für die Inanspruchnahme dieser Instrumente gesenkt worden (2.), was drittens dazu geführt hat, daß in immer mehr Bundesländern Volksbegehren und Volksentscheide auch tatsächlich stattfinden und Erfolg haben (3.). Beglaubigt und zugleich weiter vorangetrieben werden diese Entwicklungen durch die Reformvorhaben in BadenWürttemberg, Nordrhein-Westfalen und dem Saarland, auch wenn diese vorerst noch im Modus der Ankündigung verharren ${ }^{48}$.

vom 27. Januar 2008: Wiederkehr der ,,hessischen Verhältnisse“, in: ZParl. 40 (2009), S. 16 ff.; dies., Die hessische Landtagswahl vom 18. Januar 2009: der ,ewige Koch“, in: ZParl. 40 (2009), S. 358 ff.

48 Konkret sieht für das Saarland der Gesetzentwurf der Regierungskoalition aus CDU und SPD vom 17.9.2012 (LT-Drs. 15/140) eine Reihe bedeutender Erleichterungen vor. So sollen zunächst Volksbegehren künftig auch über finanzwirksame Gesetze stattfinden dürfen, soweit die finanziellen Auswirkungen 0,3\% des laufenden Haushaltsplans, bei wiederkehrenden Auswirkungen jährlich 0,5\% nicht überschreiten. Im übrigen soll die Hürde für Volksbegehren von derzeit 20\% auf 7\% abgesenkt, die Sammlungsfrist dabei von zwei Wochen auf drei Monate verlängert werden. Das Zustimmungsquorum für einfachgesetzliche Volksentscheide soll von gegenwärtig 50\% auf $25 \%$ reduziert werden. Für verfassungsändernde Volksentscheide, die bislang durch Art. 100 Abs. 4 SaarlVerf. rigoros ausgeschlossen waren, sieht der Gesetzentwurf künftig das Erfordernis einer 2/3Mehrheit der Abstimmenden bei gleichzeitiger Beteiligung von mindestens 50\% der Stimmberechtigten vor. Schließlich soll auch eine Volksinitiative eingeführt werden, für die die Unterschrift von 5.000 Stimmberechtigten erforderlich wäre. - In NordrheinWestfalen haben sich SPD und Grüne in ihrem Koalitionsvertrag (Quelle: Verantwortung für ein starkes NRW, abrufbar unter http://www.gruene-nrw.de/koalitionsvertrag.html [Zugriff 28.12.2012], S. 103), ebenfalls darauf verständigt, das Quorum für Volksbegehren ,deutlich“ abzusenken, zugleich sollen in Zukunft auch finanzwirksame Volksbegehren zugelassen werden. Ferner wird das Verfahren zur Volksinitiative grundlegend reformiert. Gleichlautend hat auch in Baden-Württemberg die grün-rote Regierungskoalition in ihrem Koalitionsvertrag (Quelle: www.spd-bw.de [Zugriff 28.12.2012], S. 59) versprochen, die Hürden für Volksbegehren „deutlich“ zu reduzieren. Diese Reduzierung dürfte sich dabei an dem orientieren, was SPD und Grüne bereits in ihrem Gesetzentwurf vom 30.8.2010 (LT-Drs. 14/6866) vorgeschlagen hatten: Absenkung des Quorums auf fünf Prozent bei gleichzeitiger Verlängerung der Sammlungsfrist auf sechs Monate und freier Unterschriftensammlung. Ferner soll eine Volksinitiative eingeführt werden; sie wird die Unterschrift von 10.000 Stimmberechtigten erfordern. 


\section{Ausbau der Mitwirkungskataloge}

Auffällig ist zuallererst, daß die schiere Zahl der dem Volk zur Verfügung stehenden direktdemokratischen Optionen gestiegen ist ${ }^{49}$. Zunächst sind die bestehenden Lücken in der klassischen Volksgesetzgebung durch Volksbegehren und Volksentscheid geschlossen worden - Schlußlicht war bekanntlich 1996 Hamburg $^{50}$. Als häufigste Erweiterung des Kanons ist die Volksinitiative oder der Bürgerantrag in den Verfassungsurkunden oft genug durch den Kleinbuchstaben „a“ als späterer Zusatz erkennbar ${ }^{51}$. Regelrechte Innovationen sind zuletzt wiederum aus Hamburg zu verzeichnen, an erster Stelle in Gestalt des fakultativen Referendums im Anschluß an die Änderung eines volksbeschlossenen Gesetzes durch die Bürgerschaft ${ }^{52}$ sowie einer flankierenden Regelung der Verbindlichkeit von Volksentscheiden auch jenseits der regelrechten Gesetzgebung ${ }^{53}$.

Auf den ersten Blick unauffällig, aber praktisch bedeutsam sind schließlich korrespondierende Maßnahmen wie Anhörungsrechte der Initiatoren ${ }^{54}$, Ansprüche auf Beratung (etwa in Bremen ${ }^{55}$ ) und - darauf wird zurückzukommen sein Kostenerstattung (so etwa in Niedersachsen und Sachsen-Anhalt ${ }^{56}$ ).

49 Wie hier Decker, Placebo (Fn. 11), S. 564 f.; C. v. Pestalozza, Volkszuständigkeiten im Grundgesetz, in: Bonner Rechtsjournal 1 (2012), S. 18 (20). O. Jung, Grundsatzfragen der direkten Demokratie, in: Kost, Demokratie (Fn. 28), S. 312 (313 f.); Rohner, Democracy (Fn. 11).

50 Vgl. nochmals v. Arnauld, Refolution (Fn. 16), S. 92 ff.

51 So in Nordrhein-Westfalen (Art. 67a LVerf.) und Rheinland-Pfalz (Art. 108a LVerf.).

52 Näher Art. 50 Abs. 4 HambVerf. und dazu nochmals v. Arnauld, Refolution (Fn. 16), S. 117 f. sowie Dressel, Demokratie (Fn. 16), S. 109 f.

53 Art. 50 Abs. 4a HambVerf. und dazu wiederum v. Arnauld, Refolution (Fn. 16), S. 119. Hintergrund war der Streit um die Privatisierung des Landesbetriebs Krankenhäuser, dazu HambVerfG, Entsch. v. 15.12.2003, Az. HVerfG 4/03, NordÖR 2004, 107 - Gesundheit ist keine Ware I u. HambVerfG, Entsch. v. 15.12.2004, Az. HVerfG 6/04, NVwZ 2005, 685 - Gesundheit ist keine Ware II sowie im Detail Rux, Demokratie (Fn. 11), S. $826 \mathrm{ff}$.

54 Sie begegnen in Berlin (Art. 61 LVerf.), Brandenburg (Art. 76 LVerf.), MecklenburgVorpommern (Art. 59 LVerf.), Niedersachsen (Art. 47 LVerf.), Nordrhein-Westfalen (§ 4 VIVBEG), Rheinland-Pfalz (Art. 60f LWG), Sachsen (Art. 71 LVerf.), Sachsen-Anhalt (Art. 80 LVerf.), Schleswig-Holstein (Art. 41 LVerf.) und Thüringen (Art. 68 LVerf.).

55 Konkret sieht $\S 8$ a VolksentscheidG vor, daß sich die Initiatoren von Bürgerschaft und Senat in ,verfassungs-, haushalts- und verfahrensrechtliche[n] Voraussetzungen und Fragen“ beraten lassen können. Vgl. dazu knapp Schefold, Neuregelung (Fn. 18), S. 143.

$56 \mathrm{Zu}$ Art. 50 Abs. 1 NdsVerf. bzw. $\S 31$ des auf Grundlage von Art. 81 Abs. 6 S-AVerf. geschaffenen VAbstG unten IV.3. 


\section{Abbau von Hürden}

Noch auffälliger und aussagekräftiger für den Paradigmenwechsel in Sachen direkter Demokratie ist zweitens die Senkung der diversen Hürden für den Volksgesetzgeber, die entweder seit den neunziger Jahren erfolgt ist ${ }^{57}$ oder in den verbleibenden Ländern dem Vernehmen nach in Angriff genommen wird ${ }^{58}$. Das sei anhand der geläufigen Stellschrauben erläutert:

Mit Händen zu greifen ist zunächst das Erodieren der Quoren für die Einleitung und Durchführung volksbeschlossener Verfahren ${ }^{59}$. Waren für das Begehrensquorum in den Ländern der alten Bundesrepublik zwanzig Prozent die Regel - dieser absurd hohe Wert, der mit dem Homogenitätsgebot des Art. 28 Abs. 1 S. 1 GG nicht mehr vereinbar sein dürfte, galt ursprünglich in sechs Ländern ${ }^{60}$ und obwaltet heute noch in Hessen und im Saarland ${ }^{61}$-, die „bloß“ zehn Prozent in Bayern der nachgerade „schweizerisch“ anmutende Ausreißer ${ }^{62}$, so dominieren heute Quoren im einstelligen Bereich: Mit einem Zwanzigstel der Wahlberechtigten orientieren sich etwa Bremen, Hamburg und Schleswig-Holstein an der aus dem Parlamentsrecht geläufigen Fünfprozenthürde als Ausweis der Erheblichkeit eines Anliegens ${ }^{63}$. Noch weiter kommt den Initiatoren Brandenburg ent-

57 Zusammenfassend zum folgenden O. Jung, Grundsatzfragen der direkten Demokratie, in: Kost, Demokratie (Fn. 28), S. 312 (323 ff.); Rux, Demokratie (Fn. 11), S. 891 ff.; Neumann, Demokratie (Fn. 8), S. 91 ff.; F. Meerkamp, Die Quorenfrage im Volksgesetzgebungsverfahren. Bedeutung und Entwicklung, 2011, S. 79 ff. u. passim sowie zuletzt $S$. Lenz, Volksgesetzgebung als „Minderheitendiktatur“?, in: ZG 28 (2013), i.E. (jeweils m.w.N.).

58 Vgl. nochmals Fn. 48.

59 Wie hier Rux, Demokratie (Fn. 11), S. 899 ff.; im Überblick Jung, Grundsatzfragen (Fn. 57), S. 326 ff. sowie B. Weixner, Direkte Demokratie in den Bundesländern. Verfassungsrechtlicher und empirischer Befund aus politikwissenschaftlicher Sicht, 2002, S. 155 ff. Siehe oben Fn. 48.

60 So ursprünglich - außer Hessen und dem Saarland - in Berlin, Bremen, NordrheinWestfalen und Rheinland-Pfalz.

61 Vgl. Art. 99 Abs. 2 S. 2 SaarlVerf. sowie Art. 124 Abs. 1 S. 1 HessVerf. In der Literatur werden die Bestimmungen überwiegend für verfassungskonform erachtet, so etwa $J$. Isensee, in: R. Wendt/R. Rixecker (Hrsg.), Verfassung des Saarlandes, 2009, Art. 99/Art. 100 Rn. 37, vgl. auch ebda. Rn. 45 ff.

62 Vgl. Rux, Demokratie (Fn. 11), S. 315 ff. - Ein erneutes Plädoyer in diese Richtung hat jetzt $M$. Möstl, Elemente direkter Demokratie als Entwicklungsperspektive, in: VVDStRL 72 (2013), i.E., Ls. 26 vorgelegt.

63 Vgl. Art. Art. 70 Abs. 1 lit. d BremVerf., Art. 50 Abs. 2 S. 8 HambVerf. und Art. 42 Abs. 1 S. 5 SHVerf. Zustimmend wie hier C. Degenhart, Volksgesetzgebungsverfahren und Verfassungsänderung nach der Verfassung des Freistaats Thüringen, in: ThürVB1. 2001, S. 201 (206) sowie Meerkamp, Quorenfrage (Fn. 57), S. 453. Ablehnend unter ausdrücklicher Kritik des Rekurses auf die Fünfprozenthürde hingegen BayVerfGH, Entsch. v. 31.3.2000, Az. Vf. 2-IX-00, BayVB1. 2000, 397 (400 f.); für verallgemeinerungsfähig und -würdig hält diese - nicht zuletzt mit bayerischen Besonderheiten begründete - 
gegen, das mit 80.000 Unterschriften ca. 3,7\% der Stimmberechtigten verlangt ${ }^{64}$. Die Mehrzahl der Länder bewegt sich zwischen fünf und zehn Prozent ${ }^{65}$.

Aussagekräftig sind diese Quoren regelmäßig erst in Kombination mit der Sammlungsfrist und der Gretchenfrage „Amtseintragung oder freie Stimmsammlung?"66 Auch hier ist das früher vorherrschende und in Bayern noch in Geltung befindliche bürokratische Abwehrmodell (zwei Wochen Sammelfrist, ausschließlich Amtseintragung ${ }^{67}$ ) ganz überwiegend von Arrangements abgelöst werden, die für die Sammlung der Unterschriften bis zu acht Monate Zeit lassen $\left(\right.$ Sachsen $\left.^{68}\right)$; die freie Stimmsammlung ist mehrheitlich zugelassen ${ }^{69}$, die Amtseintragung wird vereinzelt durch niedrigere Quoren und verlängerte Fristen privilegiert (so Thüringen ${ }^{70}$ ).

Nicht ganz so markant, aber immer noch deutlich fällt auf der zweiten Stufe des Volksentscheids der Abbau der Zustimmungs- und Beteiligungsquoren aus ${ }^{71}$.

Rechtsprechung sogar $K$. Engelken, Demokratische Legitimation bei Plebisziten auf staatlicher und kommunaler Ebene, in: DÖV 2000, S. 881 (893).

64 Siehe Art. 77 Abs. 3 S. 1 BbgVerf. und dazu H. Lieber, in: ders./S.J. Iwers/M. Ernst, Verfassung des Landes Brandenburg, 2012, Art. 77 Anm. 4; J. Platter, Volksinitiative, Volksbegehren und Volksentscheid zu Gegenständen der politischen Willensbildung (Art. 76-78 LV), in: G. Fritsch (Hrsg.), 20 Jahre Landesverfassung, 2012, S. 113 (123: 80.000 Stimmen bis 2012 nie erreicht - vgl. jetzt aber O. Jung, Dokumentation, in: L.P. Feld u.a. [Hrsg.], Jahrbuch für direkte Demokratie 2012, 2013, S. 129 [149 f.]: Volksbegehren „Nachtflugverbot“") sowie Obermann, Ländervergleich (Fn. 11), S. 247 f.

65 Weitere Nachweise bei Rux, Demokratie (Fn. 11), S. 426 f.

66 Dazu F. Wittreck, Direkte Demokratie und Verfassungsgerichtsbarkeit, in: JöR 53 (2005), S. 111 (183) sowie Weixner, Demokratie (Fn. 59), S. 164 ff. und Jürgens/Rehmet, Demokratie (Fn. 12), S. 202 ff. (jeweils m.w.N.). - Tendenziell für eine Amtseintragung bei Volksbegehren Rux, Demokratie (Fn. 11), S. 915 Fn. 3; hörenswertes Plädoyer für die fakultative Amtseintragung, die zumindest in Flächenländern den Initiatoren Mobilisierungs- und Infrastrukturlasten abnehmen könne, auch bei Meerkamp, Quorenfrage (Fn. 57), S. $521 \mathrm{f}$.

67 Vgl. Art. 71 BayVerf. i.V.m. Art. 65 Abs. 3 S. 1 (Eintragungsfrist) bzw. Art. 67, 68 (Amtseintragung) LWG i.V.m. $\S 72$ ff. LWO; kritisch Wittreck, Verfassungsgerichtsbarkeit (Fn. 66), S. 149 f. sowie S. 183 m.w.N.; für die Beibehaltung der Regelung plädiert hingegen K. Schweiger, Verfassungsgericht und Plebiszit, in: BayVB1. 2002, S. 65 (71).

68 Siehe Art. 72 Abs. 3 S. 2 SächsVerf. i.V.m. § 20 SächsVVVG und dazu W.J. Patzelt, Direkte Demokratie in Sachsen, in: Kost, Demokratie (Fn. 28), S. 246 (250).

69 Zusammenfassend m.w.N. Rux, Demokratie (Fn. 11), S. 423 ff. sowie 912 f.; Obermann, Ländervergleich (Fn. 11), S. 244 f.

70 Konkret Art. 82 Abs. 5 S. 2 ThürVerf., der vorsieht, daß bei amtlicher Eintragung innerhalb von zwei Monaten acht und bei freier Sammlung innerhalb von vier Monaten zehn Prozent der Stimmberechtigten unterzeichnet haben müssen.

71 In der Beobachtung wie hier O. Jung, Zur Problematik des Beteiligungsquorums, in: Feld u.a., Jahrbuch 2009 (Fn. 15), S. 40 (41 f.); F. Meerkamp, Die Bedeutung von Eintragungsfristen beim Volksbegehren im Rahmen der Volksgesetzgebungsverfahren in den deutschen Ländern, in: ZParl. 41 (2010), S. 579 (579 f.). 
Das schlanke und rechtspolitisch vorzugswürdige „Mehrheit entscheidet“ der Bayerischen Verfassung ${ }^{72}$ hat dabei allerdings sein Alleinstellungsmerkmal mehr oder minder behaupten können (mit Ausnahme von Hessen und Sachsen ${ }^{73}$ ); es überwiegen Zustimmungsquoren in einem Korridor von 20-25\% ${ }^{74}$, wobei Ausreißer $\left(15 \%\right.$ in Nordrhein-Westfalen ${ }^{75}$, einmal mehr absurde $50 \%$ im Saarland $^{76}$ ) $\mathrm{zu}$ verzeichnen sind. Ein Beteiligungsquorum findet sich nur noch in RheinlandPfalz (auch hier $25 \%{ }^{77}$ ). Zum Zwecke des erhöhten Bestandsschutzes der Verfassungsurkunde, der in allen Verfassungen normtextlich ${ }^{78}$ oder richterrechtlich ${ }^{79}$ gewährleistet ist, begegnen schließlich Zustimmungsquoren - statt aller sei Bremen erwähnt, das für die Verfassungsänderung ein solches Quorum von $50 \%$ ansetzt $^{80}$.

Deutlich weniger Bewegung ist hingegen in die Ausschlußtatbestände gekommen $^{81}$, an erster Stelle das sog. Finanztabu in seinen historisch bedingten unterschiedlichen Ausprägungen ${ }^{82}$. Die explizite Zulassung „finanzwirksamer“

72 Art. 2 Abs. 2 BayVerf. und dazu J.F. Lindner, in: ders./M. Möstl/H.A. Wolff, Verfassung des Freistaates Bayern, 2009, Art. 2 Rn. 10 ff.

73 Art. 124 Abs. 3 HessVerf. bzw. Art. 72 Abs. 4 SächsVerf.; vgl. dazu F.K. Schonebohm, in: G.A. Zinn/E. Stein (Hrsg.), Verfassung des Landes Hessen, Art. 124 (1984), Erl. VIII 4 sowie U. Fastenrath, Staatsvolk, Staatsbürgerschaft, Minderheitenschutz, in: C. Degenhart/C. Meissner (Hrsg.), Handbuch der Verfassung des Freistaates Sachsen, 1997, $\S 4$ Rn. 26; vgl. Obermann, Ländervergleich (Fn. 11), S. 245.

74 Zusammenfassend Obermann, Ländervergleich (Fn. 11), S. 245; Jürgens/Rehmet, Demokratie (Fn. 12), S. 202 ff.

75 Siehe Art. 68 Abs. 4 S. 2 NWVerf. und dazu Mann (Fn. 30), Art. 68 Rn. 44; Günther (Fn. 30), Art. 68 Rn. 23; Grawert (Fn. 30), Art. 68 Anm. 3.

76 Siehe Art. 100 Abs. 3 SaarlVerf. - Die Regelung verteidigt Isensee (Fn. 61), Art. 99/Art. 100 Rn. 5, 8, 10. Auch hier dürfte freilich gelten, daß sie in ihrer Verhinderungslogik demokratische Grundsätze berührt und daher mit Art. 28 Abs. 1 S. 1 GG nicht vereinbar ist; von einem ,in der Praxis unerreichbaren“ Quorum spricht Decker, Placebo (Fn. 11), S. 568.

77 Dazu nur D. Franke, in: Grimm/Caesar, Verfassung (Fn. 19), Art. 109 Rn. 22.

78 Überblick m.w.N. bei Jürgens/Rehmet, Demokratie (Fn. 12), S. 206 f.

79 In Bayern geht ein Zustimmungsquorum von 25 Prozent lediglich auf die Entscheidung des BayVerfGH v. 17.9.1999, Az. Vf. 12-VIII-98, Vf.14-VII-98, Vf.15-VII-98, BayVBl. 1999, 719 zurück. Dazu kritisch O. Jung, 50 Jahre verfassungswidrige Volksgesetzgebung in Bayern?, in: BayVB1. 1999, S. 417 ff. sowie Wittreck, Verfassungsgerichtsbarkeit (Fn. 66), S. 138 ff., $147 \mathrm{f}$.

80 Vgl. Art. 72 Abs. 2 BremVerf. und dazu Schefold, Neuregelung (Fn. 18), S. 136, 148.

81 Instruktiv zur kommunalen Ebene T. Weßels, Rechtliche Beurteilung der Ausnahmetatbestände und deren Umgehungsgefahr bei Bürgerbegehren und Bürgerentscheid, 2012, S. $225 \mathrm{ff}$.

82 Zusammenfassend O. Jung, Das Finanztabu bei der Volksgesetzgebung, in: Der Staat 38 (1999), S. 41 ff.; J. Krafczyk, Der parlamentarische Finanzvorbehalt bei der Volksgesetzgebung, 2005, S. 25 ff.; M. Klatt, Die Zulässigkeit des finanzwirksamen Plebiszits, in: Der Staat 50 (2011), S. 3 ff. sowie C. Waldhoff/H. v. Aswege, Direkte Demokratie und Staatsfinanzkrise - Abschaffung der Finanztabus als Ausweg?, in: Feld u.a., Jahrbuch 
Volksbegehren (die dann konsequenterweise mit einem Deckungsvorschlag versehen werden müssen) in Bremen ist insofern ein Solitär ${ }^{83}$; die weniger weitreichenden Vorschriften in Berlin und Hamburg, die lediglich das „Landeshaushaltsgesetz“ (Art. 61 Abs. 2 BerlVerf.) bzw. „Haushaltspläne“ (Art. 50 Abs. 1 S. 2 HambVerf.) ausnehmen, sind in ihrer Auslegung nicht von ungefähr umstritten ${ }^{84}$. In Sachsen hat schließlich das Landesverfassungsgericht durch eine einschränkende Auslegung der einschlägigen Ausschlußklausel ähnlich viel Raum wie in Bremen geschaffen ${ }^{85}$.

\section{Ankunft im Alltag}

Damit sind wir schon beim Praxisvollzug. Mit der markanten Ausnahme Bayerns haben Instrumente der direkten Demokratie in der Bundesrepublik lange Jahre eine „Kümmerexistenz am Rande der [politischen] Gesellschaft“ gefristet $^{86}$. Das hat sich ebenso markant geändert. Zwar gibt es nach wie vor eine Reihe von Bundesländern, die - aus angebbaren Gründen - noch ganz ohne Volksbegehren oder Volksentscheid sind (es drängt sich einmal mehr das Saarland als Beispiel auf $^{87}$ ) oder im niedrigen einstelligen Bereich verharren ${ }^{88}$. Mit Berlin und Ham-

$$
2011 \text { (Fn. 15), S. } 9 \text { (14 ff.). }
$$

83 Siehe Art. 70 Abs. 2 S. 2 BremVerf. und dazu Schefold, Neuregelung (Fn. 18), S. 143 ff.

84 Berlin: Hier hat der Verfassungsgerichtshof die Korrektur seiner Rechtsprechung durch den verfassungsändernden Gesetzgeber immerhin akzeptiert; vgl. BerlVerfGH, Entsch. v. 22.11.2005, Az. 35/04, juris - Berliner Bankenskandal mit BerlVerfGH, Entsch. v. 6.10.2009, Az. 63/08 u. 143/08, LKV 2009, 514 - Kitakinder und dazu M. Hellriegel, Entscheidungsanmerkung, in: LKV 2009, S. 516 (516 f.). Die (zähneknirschende) Rechtsprechungswende und ihren Kontext schildern näher Pestalozza, Weg (Fn. 42), S. 307 ff. sowie O. Jung, Die Reform der direkten Demokratie in Berlin 2006 (Teil II), in: L.P. Feld/P.M. Huber/H.-J. Lauth/ders./F. Wittreck (Hrsg.), Jahrbuch für direkte Demokratie 2012, i.E. - Hamburg: Für die bloße Festschreibung des status quo v. Arnauld, Refolution (Fn. 16), S. 105; für eine weite Auslegung M. Klatt, Auslegung und Verfassungsmäßigkeit des Art. 50 Abs. 1 S. 2 HmbVerf, in: NordÖR 2010, S. 482 (483); für grundgesetzwidrig halten die Norm schließlich (ohne durchgreifende Argumente) P. Selmer/L. Hum$m e l$, Zulässige Volksentscheide über alle haushaltsplanexternen Haushaltsangelegenheiten?, in: NordÖR 2009, S. 137 (140 ff.).

85 Vgl. SächsVerfGH, Entsch. v. 11.7.2002, Az. Vf. 91-VI-01, SächsVB1. 2002, 236 und dazu P. Neumann, Durchbruch bei der Ausgestaltung der Volksgesetzgebung, in: SächsVB1. 2002, S. 229 ff.; D. Zschoch, Volksgesetzgebung und Haushaltsvorbehalt, in: NVwZ 2003, S. 438 ff.; J. Kertels/S. Brink, Quod licet jovi - Volksgesetzgebung und Budgetrecht, in: NVwZ 2003, 434 (436 ff.); Wittreck, Verfassungsgerichtsbarkeit (Fn. 66), S. $170 \mathrm{ff}$.

86 In Anlehnung an BVerfGE 26, 141 (158) - Richterbesoldung I.

87 Neben dem Saarland erfreut sich noch Baden-Württemberg praktisch direktdemokratischer Jungfräulichkeit auf Landesebene (zumindest dann, wenn man die „von oben“ initiierte Abstimmung über „Stuttgart 21“ nicht berücksichtigt); Daten bei Mehr Demokratie 
burg haben bezeichnenderweise zwei Stadtstaaten dicht zu Bayern aufgeschlos$\operatorname{sen}^{89}$, und Volksbegehren oder zumindest Versuche, sie zu starten, sind praktisch in allen Bundesländern zu verzeichnen ${ }^{90}$.

Diese Entwicklung - die mit hohen Zustimmungsraten der Bürger einhergeht $^{91}$ - gilt es nun zunächst einmal vorurteilsfrei, aber auch kritisch zu beobachten. Das dürfte um so wichtiger sein, als die deutsche Diskussion über direkte Demokratie und ihre Auswirkungen bislang durch eine eminente Empirieaversion gekennzeichnet ist (dies gilt zumindest für die staatsrechtliche Debatte ${ }^{92}$ ).

\section{Direkte Demokratie in den Ländern: Offene Fragen}

Bisher ergibt sich danach - zumindest für den, der (mehr) direkte Demokratie nicht grundsätzlich als Irrweg oder als Zumutung betrachtet - ein durchaus erfreuliches Bild. In einem dritten Schritt gilt es, hanseatisch nüchtern Wasser in den Wein zu gießen. Denn es fällt zunächst auf, daß der geschilderte „Siegeszug“ die volksbeschlossene Verfassungsänderung oft genug ausspart (1.). Zweitens ist darzulegen, daß in dem Maße, in dem die direkte Demokratie vom Rande in die Mitte der politischen Arena rückt, die Frage der praktischen Konkurrenz oder Konkordanz von Volks- und Parlamentsgesetzgeber dringlich wird (2.). Schließlich zeichnen sich gerade in der jüngsten Entwicklung Probleme der Finanzierung bzw. des effektiven Rechtsschutzes direktdemokratischer Initiativen $\mathrm{ab}(3$.$) .$

e.V. (Hrsg.), Drittes Volksentscheids-Ranking, 2010, S. 42 f.

88 Dies gilt für Rheinland-Pfalz, Hessen oder Mecklenburg-Vorpommern. Daten abermals bei Mehr Demokratie, Ranking (Fn. 87), S. 36 ff.

89 Vgl. Mehr Demokratie, Ranking (Fn. 87), S. 28 f. Siehe auch M. Efler/C. Posselt, Direkte Demokratie in Berlin, in: Heußner/Jung, Demokratie ${ }^{2}$ (Fn. 8), S. 285 ff.; sowie O. Jung, Dokumentation, in: Feld u.a., Jahrbuch 2011 (Fn. 15), S. 127 (131 ff.); zu Hamburg noch Dressel, Demokratie (Fn. 16), S. 107.

90 Nochmals Mehr Demokratie, Ranking (Fn. 87), S. 28 ff.

91 Vgl. L.P. Feld/Z. Hessami/L. Reil, Direkte Demokratie in der Bundesrepublik Deutschland? - Ergebnisse einer Umfrage zur Einführung direkter Volksrechte auf Bundesebene, in: Feld u.a., Jahrbuch 2010 (Fn. 1), S. 107 (115 ff.); unterstrichen auch von Möstl, Elemente (Fn. 62), Ls. 11.

92 Wie hier Eder, Demokratie (Fn. 11), S. 22. 
Zwar ist der ändernde Zugriff auf die Verfassung dem Volk nur in Hessen ${ }^{93}$ und dem Saarland ${ }^{94}$ explizit versagt (in Hessen teilweise kompensiert durch das bereits erwähnte obligatorische Referendum nach Parlamentsbeschlu $\beta^{95}$ ). Gleichwohl ist zu beobachten, daß der Versuch einer „vollplebiszitären“ Verfassungsänderung ${ }^{96}$ selbst bei Einhaltung der formalen Regeln zum erhöhten Bestandsschutz eine gefahrgeneigte Arbeit darstellt oder - präziser gewendet - den besonderen Argwohn der politischen Klasse einschließlich der Verfassungsgerichtsbarkeit auf sich zieht (oder zumindest gezogen hat).

Nun mag man über die politische Klugheit der um das Jahr 2000 maßgeblich vom Verein „Mehr Demokratie“ initiierten Volksbegehren füglich streiten, die in mehreren Bundesländern substantielle Modifikationen des direktdemokratischen Instrumentariums anstoßen sollten ${ }^{97}$. Aus dem Abstand von zehn Jahren lassen sich nunmehr zwei Befunde festhalten:

Erstens: Diese Volksbegehren sind damals nicht an der Urne, sondern am Richterstuhl gescheitert, genauer gesagt an einem tiefsitzenden Verteidigungsreflex der Rechtsprechung zugunsten des verfassungsrechtlichen status quo resp. eines vermeintlichen Vorrangs der repräsentativen Demokratie. Der Verfasser hat an anderer Stelle zu erläutern versucht, daß zumindest einige der dabei ins Feld geführten Argumente Niklas Luhmanns Ausführungen zur komplexitätsre-

93 Nach Art. 123 Abs. 2 HessVerf. kommt eine Verfassungsänderung durch Beschluß des Landtags mit der Mehrheit der gesetzlichen Mitgliederzahl zustande, sofern sie anschließend im Referendum vom Volk bestätigt wird; näher U. Dreßler, Direkte Demokratie in Hessen, in: Kost, Demokratie (Fn. 28), S. 133 (137). Die herrschende Auffassung, daß damit zugleich eine ,vollplebiszitäre“ Verfassungsänderung ausgeschlossen sei (statt aller F.K. Schonebohm, in: Zinn/Stein, Verfassung [Fn. 73], Art. 124 [1984], Erl. II 2), wird mit gewichtigen entstehungsgeschichtlichen Argumenten in Frage gestellt von $O$. Jung, Volksbegehren auf Verfassungsänderung in Hessen und Nordrhein-Westfalen?, in: KritV 76 (1993), S. 14 (21).

94 Art. 101 Abs. 2 S. 1 SaarlVerf. sieht nur die Änderung durch Zweidrittelmehrheit im Landtag vor; vgl. im Gegenschluß auch Art. 99 Abs. 1 S. 1 SaarlVerf. - Dazu J. Wohlfahrt, Direkte Demokratie im Saarland, in: Kost, Demokratie (Fn. 28), S. 228 (229) sowie Isensee (Fn. 61), Art. 99/Art. 100 Rn. 26.

95 Vgl. oben Fn. 38.

96 Dies in Anlehnung an J. Isensee, Verfassungsreferendum mit einfacher Mehrheit, 1999, S. 40.

97 Ein klassischer taktischer Fehler dürfte insbesondere der Versuch gewesen sein, die - in der Tat völlig verfehlte - Rechtsprechung des Bayerischen Verfassungsgerichtshofs zum notwendigen Quorum bei einer vollplebiszitären Verfassungsänderung durch einen weiteren Volksentscheid zu korrigieren: näher Wittreck, Verfassungsgerichtsbarkeit (Fn. 66), S. $138 \mathrm{ff}$. 
duzierenden Funktion des Rechts ${ }^{98}$ vielleicht ein wenig zu wörtlich genommen haben ${ }^{99}$.

Zweitens: So brachial das historische Scheitern, so fulminant bereits der mittelfristige Erfolg. Denn im Konsens mit den Repräsentanten sind nur wenige Jahre später namentlich in Thüringen sowie hier in Bremen Verfassungsreformen verabschiedet worden ${ }^{100}$, die zentrale Forderungen der damaligen Volksbegehren aufgreifen: Senkung der Quoren ${ }^{101}$, freie Stimmsammlung ${ }^{102}$, in Bremen gar finanzwirksame Volksgesetzgebung ${ }^{103}$ (Bayern ist sich hingegen treu geblieben $\left.^{104}\right)$.

N. Luhmann, Das Recht der Gesellschaft, 1993, S. 225 f.; vgl. dazu nur T. Raiser, Grundlagen der Rechtssoziologie, 4. Aufl. 2007, S. 122 sowie A. Büllesbach, Systemtheorie im Recht, in: A. Kaufmann/W. Hassemer/U. Neumann (Hrsg.), Einführung in die Rechtsphilosophie und Rechtstheorie der Gegenwart, 8. Aufl. 2011, S. 428 (441 ff.).

99 Eingehend und m.w.N. Wittreck, Verfassungsgerichtsbarkeit (Fn. 66), S. 111 ff. sowie ders., Direkte Demokratie und Rechtsprechung - ein gestörtes Verhältnis?, in: Heußner/Jung, Demokratie ${ }^{2}$ (Fn. 8), S. 397 ff. Vgl. noch O. Jung, Direkte Demokratie - vom Kopf auf die Füße gestellt, in: LKV 2003, S. 308 ff.

100 Zusammenfassend zur Neuregelung in Thüringen C. Degenhart, Direkte Demokratie in den ostdeutschen Bundesländern, in: Heußner/Jung, Demokratie ${ }^{2}$ (Fn. 8), S. 271 (273, 277 f.); zu Bremen Schefold, Neuregelung (Fn. 18), S. 135 ff.; vgl. auch S. Storr, Volksbegehren sperrt Parlamentsgesetz?, in: Feld u.a., Jahrbuch 2009 (Fn. 15), S. 285 ff.

101 Konkret: In Thüringen von 14\% der Stimmberechtigten auf acht bzw. zehn Prozent (Zustimmungsquorum im Volksbegehren, vgl. Art. 82 Abs. 5 ThürVerf) resp. von einem Drittel der Stimmberechtigten auf ein Viertel (Zustimmungsquorum im Volksentscheid, vgl. Art. 82 Abs. 7 S. 3 ThürVerf.) sowie von 50\% der Stimmberechtigten auf $40 \%$ (Zustimmungsquorum im verfassungsändernden Volksentscheid, vgl. Art. 83 Abs. 2 S. 2 ThürVerf.). In Bremen von zehn Prozent der Stimmberechtigten auf fünf Prozent (Zustimmungsquorum im Volksbegehren, vgl. Art. 70 Abs. 1 S. 1 lit. d BremVerf.) und von einem Viertel der Stimmberechtigten auf ein Fünftel (Zustimmungsquorum im Volksentscheid, vgl. Art. 72 Abs. 1 BremVerf.).

102 Siehe Art. 82 Abs. 5 S. 1 ThürVerf., der den Initiatoren die Wahl zwischen beiden Varianten läßt.

103 Vgl. oben Fn. 18.

104 Mit der Folge, daß hier auch mehrere „einfache“ Volksbegehren etwa wegen Verstoßes gegen das „Finanztabu“ (Art. 73 BayVerf.) vom Verfassungsgerichtshof gestoppt worden sind, so beispielsweise BayVerfGH, Entsch. v. 4.4.2008, Az. Vf. 8-IX-08, NVwZ-RR 2008, 719 - Transrapid und Entsch. v. 17.11.1994, Az. Vf. 96 IX-94, Vf. 97-IX-94, BayVBl. 1995, 173 - Volksbegehren Schulgesetz. - Zuletzt hat der VerfGH mit Entsch. v. 22.10.2012, Az. Vf. 57-IX-12, dokumentiert in: F. Wittreck, Ausgewählte Entscheidungen zur direkten Demokratie, in: Feld u.a., Jahrbuch 2012 (Fn. 64), im Fall eines Volksbegehrens gegen die Erhebung von Studiengebühren einen Verstoß gegen Art. 73 BayVerf. jedoch überraschend verneint. Kritisch zur weiten Auslegung von Art. 73 BayVerf. C. Degenhart, Volksbegehren und Volksentscheide "über den Haushalt" - zur Auslegung des Art. 73 BV, in: BayVBl. 2008, S. 453 ff. sowie Schweiger, Verfassungsgericht (Fn. 67), S. 70; zustimmend hingegen M. Möstl, in: Lindner/Möstl/Wolff, Verfassung (Fn. 72), Art. 73 Rn. 6. 
Nur: Wo bleibt der Aufschrei? Wo die Klage? Zur Erinnerung: Wir reden über Reformelemente, denen um 2000 herum noch schwarz auf weiß bescheinigt worden ist, sie verstießen entweder gegen die Ewigkeitsklausel der Landesverfassung (so in Thüringen ${ }^{105}$ ) oder gegen das Homogenitätsgebot des Art. 28 Abs. $1 \mathrm{GG}$ (so in Bremen ${ }^{106}$ ), seien also schlechthin undemokratisch und dürften niemals verwirklicht werden. Hat hier womöglich ein Lernprozeß eingesetzt?

\section{2. „Prävalenz“ und Konterlegislatur}

Lassen wir die Debatten und Argumente der Vergangenheit ruhen und wenden uns den Problemen der Zukunft zu. Akzeptiert man nämlich die Prognose, daß Volksgesetzgebung deutlich häufiger werden wird, so muß man zu dem Schluß kommen, daß ein zentrales Dilemma direkter Demokratie von jetzt an das ganz praktische Verhältnis von Volks- und Parlamentsgesetzgeber sein wird ${ }^{107}$. Damit ist das Phänomen der sog. Konterlegislatur angesprochen ${ }^{108}$, die hier als Oberbegriff für gleich zwei Konfliktkonstellationen fungieren soll, die beide aus der systemischen Schwerfälligkeit der Volksgesetzgebung resultieren. Zunächst können sich Parlament wie Exekutive versucht sehen, schon während des plebiszitären Verfahrens „Fakten zu schaffen“, indem durch Gesetzesänderungen oder sonstige Verfügungen etwa einem Volksbegehren das rechtliche oder sachliche Substrat entzogen wird (man denke hier an den Streit über die Reform der Thüringer Gemeindeordnung ${ }^{109}$ ). Auch nach dem Volksentscheid bleibt zweitens die Option, das volksbeschlossene Gesetz bei erster Gelegenheit zu ändern oder ganz

105 Vgl. ThürVerfGH, Urt. v. 19.9.2001, Az. VerfGH 4/01, LVerfGE 12, 405 (432 ff.). Zustimmend Isensee (Fn. 61), Art. 100 Rn. 46; kritisch etwa M. Sachs, Ewigkeitsgarantie für Grenzen der Volksgesetzgebung?, in: LKV 2002, S. 249 (250 ff.): ,gefühlsmäßig anmutende[n] Abwehrhaltung“ (Zitat S. 250); S. Jutzi, Anmerkung zum Urteil des Thüringer VerfGH vom 19. September 2001, in: NJ 2001, S. 645 (646): „Zementierungsrechtsprechung“, die das Volk ,eher als Gefahr“" wahrnehme.

106 BremStGH, Urteil v. 14.2.2000, Az. St 1/1998, NVwZ-RR 2001, S. 1 (2 ff.). Zustimmend $J$. Isensee, Volksgesetzgebung - Vitalisierung oder Störung der parlamentarischen Demokratie, in: DVB1. 2001, S. 1161 (1166 ff.); kritisch hingegen O. Jung, Direkte Demokratie nach Schweizer Art in Deutschland verfassungswidrig? in: KritV 84 (2001), S. 24 (52): „Überängste“ schienen mitunter die „disziplinierte Arbeit der Verfassungsdogmatik ersetzt zu haben“, teils sei das Urteil gar ,peinlich“.

107 Vgl. zum folgenden die neuesten Darstellungen von F. Wittreck, Einleitung: Direkte und repräsentative Demokratie zwischen Konkurrenz und Konkordanz, in: ders. (Hrsg.), Volks- und Parlamentsgesetzgeber: Konkurrenz oder Konkordanz?, 2012, S. 9 (13 ff.) sowie M. Martini, Wenn das Volk (mit)entscheidet ..., 2011, S. $38 \mathrm{ff}$.

108 Terminus bei Jung, Konterlegislatur (Fn. 16), S. 427 (Definition ebda., S. 428).

109 Dokumentiert in: Wittreck, Parlamentsgesetzgeber (Fn. 107); vgl. auch Storr, Volksbegehren (Fn. 100), S. 285 ff. 
aufzuheben ${ }^{110}$ - sie lockt namentlich dann, wenn der nächste Wahltermin noch beruhigend weit weg ist (an Beispielen wäre kein Mangel ${ }^{111}$ ).

Die Versuche, dieses Konkurrenzverhältnis zweier dem Grunde nach gleichrangiger Gesetzgeber dogmatisch zu domestizieren, sind entweder nicht überzeugend oder ineffektiv ${ }^{12}$. Jegliche Spekulation über eine „Präponderanz“ oder „Prävalenz“ eines der beiden Akteure ${ }^{113}$ ist zunächst disziplinär der politischen Theologie, nicht aber der Rechtswissenschaft zuzuordnen (zudem heuristisch wertlos, weil sie keine handhabbaren Abstufungen bereithält) ${ }^{114}$. Und die Verfassungsorgantreue steht nicht nur normtextlich auf schwachen Füßen ${ }^{115}$, sondern hat auch bislang im Ergebnis nie dazu geführt, dem deutlich agileren Parlamentsgesetzgeber wirklich Grenzen zu ziehen ${ }^{116}$. Vorzugswürdig dürfte vor diesem Hintergrund die Lösung durch den jeweiligen verfassungsändernden Gesetzgeber sein, der sich an der (fakultativen) Referendumslösung in Hamburg

110 Aus der Literatur: M. Borowski, Parlamentsgesetzliche Änderungen volksbeschlossener Gesetze, in: DÖV 2000, S. 481 ff.; M. Rossi/S.-C. Lenski, Treuepflichten im Nebeneinander von plebiszitärer und repräsentativer Demokratie, in: DVB1. 2008, S. 416 (417).

111 Außer der Rückkehr zur sog. neuen Rechtschreibung in Schleswig-Holstein (dazu $B$. Kliegis/U.G. Kliegis, Der Volksentscheid über die Rechtschreibreform in SchleswigHolstein 1998, in: Heußner/Jung, Demokratie1 [Fn. 7], S. 287 ff.) ist hier namentlich an die verschiedenen Hamburger Fälle zu denken: Näher v. Arnauld, Refolution (Fn. 16), S. 96 ff. sowie Rux, Demokratie (Fn. 11), S. 826 ff.

112 Ähnlich zurückhaltend v. Arnauld, Refolution (Fn. 16), S. 116 f.

113 Klassisch E.-W. Böckenförde, Mittelbare/repräsentative Demokratie als eigentliche Form der Demokratie, in: G. Müller (Hrsg.), Staatsorganisation und Staatsfunktionen im Wandel. Festschrift für Kurt Eichenberger, 1982, S. 301 ff. Für einen - im einzelnen unterschiedlich ausgestalteten - normativen Vorrang des Repräsentativsystems ferner $P$. Badura, Die parlamentarische Demokratie, in: J. Isensee/P. Kirchhof (Hrsg.), Handbuch des Staatsrechts der Bundesrepublik Deutschland, Bd. II, 3. Aufl. 2004, § 25 Rn. 35, 40, 44, 60; zuletzt P.M. Huber, Aktuelle Fragen der direkten Demokratie im Freistaat Thüringen, in: Wittreck, Parlamentsgesetzgeber (Fn. 107), S. 153 (168 ff.) sowie Möstl, Elemente (Fn. 62), Ls. 8.

114 Prononciert für einen normativen Gleichrang beider Akteure Rossi/Lenski, Treuepflichten (Fn. 110), S. 418 f.; v. Arnauld, Refolution (Fn. 16), S. 116; H. Dreier/F. Wittreck, Repräsentative und direkte Demokratie im Grundgesetz, in: Feld u.a., Jahrbuch 2009 (Fn. 15), S. 11 (16 ff.) sowie H. Meyer, Antragsschrift, in: Wittreck, Parlamentsgesetzgeber (Fn. 107), S. 27 (30 f.).

115 Grundsätzlich kritisch zur ungeschriebenen Figur der „Bundestreue“ jetzt $F$. Wittreck, Bundestreue, in: I. Härtel (Hrsg.), Handbuch Föderalismus, Bd. I, 2012, § 18 Rn. 3 ff. (speziell zur Verfassungsorgantreue Rn. 53).

116 Vgl. namentlich die einschlägigen Entscheidungen des Hamburgischen Verfassungsgerichts: HbgVerfG, Entsch. v. 15.12.2004, Az. HVerfG 6/04, NVwZ 2005, 685 (688 f.) Gesundheit ist keine Ware II; HVerfG, Entsch. v. 27.4.2007, Az. HVerfG 4/06, NordÖR 2007, 301 (304 f.) - Wahlrechtsänderungsgesetz; siehe zu den konkreten Schlußfolgerungen ferner Storr, Volksbegehren (Fn. 100), S. 289 ff.; Martini, Volk (Fn. 107), S. 62 ff., 84 ff. sowie Huber, Fragen (Fn. 113), S. 176 ff. 
orientieren könnte ${ }^{117}$. Sie hebt den politischen Preis der parlamentarischen Änderung eines volksbeschlossenen Gesetzes auf ein Niveau, das Eingriffe aus Gedankenlosigkeit oder Frivolität ausschließt, nachvollziehbaren Gemeinwohlanliegen aber hinreichend Raum läßt ${ }^{118}$.

\section{Finanzierung und Rechtsschutz}

Ähnliche Vorbilder finden sich inzwischen auch für zwei weitere Problemkreise, die abschließend kurz umrissen werden sollen: Die Finanzierung von plebiszitären Instrumenten ${ }^{119}$ sowie der Rechtsschutz, namentlich während des laufenden Verfahrens (oder sollte man „des stockenden“ sagen?) ${ }^{120}$.

Beginnen wir mit dem Geld. Namentlich aus den USA ist sattsam bekannt, daß direkte Demokratie, sobald sie einmal als praktikable politische Option zur Durchsetzung von Positionen erkannt worden ist, auch finanzkräftige Akteure anziehen kann $^{121}$; die resultierenden Praktiken weisen zum Stimmenkauf teils nur noch graduelle Unterschiede auf ${ }^{122}$. Vor diesem Hintergrund gibt es gleich zwei gute Argumente dafür, über eine öffentliche (Mit-)Finanzierung direktdemokratischer Instrumente nachzudenken, sofern sie bestimmte Relevanzschwellen denn überwunden haben. Das erste zielt darauf, Initiativen wenigstens ein Stück weit unabhängig von potenten organisierten Interessen zu machen ${ }^{123}$. Das zweite fordert Gleichbehandlung mit dem primär parteipolitisch dominierten Re-

117 Vgl. oben Fn. 16; wie hier v. Arnauld, Refolution (Fn. 16), S. 117.

118 Nicht empfehlenswert ist hingegen die etwa in Kalifornien begegnende Lösung eines obligatorischen Referendums. Vgl. dazu nur H.K. Heußner, Die Krise Kaliforniens - Die Schuld der direkten Demokratie?, in: Feld u.a., Jahrbuch 2011 (Fn. 15), S. 175 (179 f., 230 und passim).

119 Dazu eingehend aus eidgenössischer wie rechtsvergleichender Sicht M. Caroni, Geld und Politik, 2009.

120 Zusammenfassend Przygode, Rechtsprechung (Fn. 12); Wittreck, Verfassungsgerichtsbarkeit (Fn. 66), S. 111 ff.; ders., Rechtsprechung (Fn. 99), S. 397 ff.; zuletzt T. Mann, Rechtsschutz bei Bürger- und Volksbegehren in Deutschland, in: P. Neumann/D. Renger (Hrsg.), Sachunmittelbare Demokratie im interdisziplinären und internationalen Kontext 2008/2009, 2010, S. 79 ff.

121 Instruktiv H.K. Heußner, Direkte Demokratie in den US-Gliedstaaten im Jahr 2008, in: Feld u.a., Jahrbuch 2009 (Fn. 15), S. 165 (196 ff.); vgl. ferner D.A. Smith, US States, in: K. Gilland Lutz/S. Hug (Hrsg.), Financing Referendum Campaigns, Basingstoke u.a. 2010, S. 39 (50 ff.); zur institutionellen Absicherung siehe Przygode, Rechtsprechung (Fn. 12), S. 451.

122 Dezidiert kritisch daher H.P. Hertig, Sind Abstimmungserfolge käuflich?: Elemente der Meinungsbildung bei eidgenössischen Abstimmungen, in: Schweizerisches Jahrbuch für Politische Wissenschaft 22 (1982), S. 35 (56): „Eine gute Kaufchance ist [...] unseres Erachtens im allgemeinen gegeben."

123 In diese Richtung auch Heußner, Gliedstaaten (Fn. 121), S. 197 f. 
präsentativprozeß ein ${ }^{124}$; will man die unlängst festgestellte „Parteiendominanz direkter Demokratie ${ }^{\text {(125 }}$ nicht noch verstärken und korrespondierende Abhängigkeitsverhältnisse schaffen, so bietet sich auch hier eine finanzielle Grundausstattung an ${ }^{126}$.

Mustert man vor diesem Hintergrund die Landschaft in den Bundesländern, so sehen mittlerweile sechs Landesverfassungen oder Umsetzungsgesetze Regelungen zur Erstattung der Kosten von Volksbegehren bzw. der Kosten des Abstimmungskampfes vor (Hamburg, Niedersachsen, Schleswig-Holstein, Sachsen, Sachsen-Anhalt und Thüringen) ${ }^{127}$. Sie differieren im Detail; drei Beispiele mögen genügen: In Sachsen etwa erhalten die Vertrauenspersonen für zehn Unterschriften im Volksbegehren $0,51 €$, für $100 \mathrm{Ja}$-Stimmen im Volksentscheid noch einmal $1,02 €^{128}$. Hamburg zahlt ohne Differenzierung $0,10 €$ für jede gültige JaStimme im Volksentscheid ${ }^{129}$; Thüringen wiederum vergütet Unterschriften im Begehren mit $0,15 €$, Ja-Stimmen im Volksentscheid mit $0,075 €^{130}$. Stets kommen Deckelungsmechanismen zum Tragen ${ }^{131}$. Im Sinne der Chancengleichheit und funktionsgerechten Ausgestaltung der direkten Demokratie dürften solche Regelungen vielleicht nicht verfassungsrechtlich geboten, aber angeraten $\operatorname{sein}^{132}$.

Zuletzt zum Rechtsschutz. Er ist derzeit stark fragmentiert, lückenhaft und noch dazu unübersichtlich ${ }^{133}$. In den meisten Bundesländern existieren nebenei-

124 Dafür dem Grunde nach Przygode, Rechtsprechung (Fn. 12), S. 459, ohne allerdings eine analoge Anwendung der Vorschriften zur Parteienfinanzierung für möglich zu halten; ebenso auch Neumann, Demokratie (Fn. 8), S. 654.

125 E.-C. Hornig, Die Parteiendominanz direkter Demokratie in Westeuropa, 2011.

126 So auch Jung, Grundsatzfragen (Fn. 57), S. 332 f.; Przygode, Rechtsprechung (Fn. 12), S. 453 sowie - trotz an sich kritischer Grundhaltung zur direkten Demokratie - Schmitz, Volksgesetzgebung (Fn. 11), S. 734.

127 Zusammenfassend Przygode, Rechtsprechung (Fn. 12), S. 451 ff. (noch zur älteren Rechtslage); Jürgens/Rehmet, Demokratie (Fn. 12), S. $207 \mathrm{ff}$.

128 Für das Volksbegehren regelt dies $\S 24$ Abs. 2 SächsVVVG, die Erstattung für den Volksentscheid findet sich in $\S 47$ Abs. 2 SächsVVVG. Zu diesem Gesetz vgl. V. Krieg, Die Volksgesetzgebung im Freistaat Sachsen, in: ZG 11 (1996), S. 314 ff., zur Kostenregelung speziell auf S. 320 .

129 Dies folgt aus $\S 30$ a Abs. 2 HVAbstG.

$130 \S 29$ Abs. 2 S. 2 ThBVVG bzgl. des Volksbegehrens, für den Entscheid: § 29 Abs. 3 S. 2.

131 Konkret $\S 30$ a Abs. 2 a.E. HambVAbstG; eine relative Begrenzung auf die für den Erfolg notwendigen Stimmen findet sich in $\S 29$ Abs. 2 S. 3, Abs. 3 S. 3 ThBVVG.

132 Ähnlich O. Jung, Welche Regeln empfehlen sich bei der Einführung von Volksbegehren und Volksentscheid (Volksgesetzgebung) auf Bundesebene?, in: Evangelische Akademie Hofgeismar/Stiftung MITARBEIT (Hrsg.), Direkte Demokratie in Deutschland. Handreichungen zur Verfassungsdiskussion in Bund und Ländern. Mit Entwürfen zur Einführung von Volksbegehren und Volksentscheid auf Bundesebene (,Hofgeismarer Entwurf“), 1991, S. 19 (51 ff.); vgl. weiterhin Neumann, Demokratie (Fn. 8), S. 823: ,sachgerecht“; explizite Kritik an einer Erstattung wird - soweit ersichtlich - nicht geäußert.

133 Kritisch daher jeweils zu den einzelnen Phasen des direktdemokratischen Prozesses Przygode, Rechtsprechung (Fn. 12), S. 103 ff., 175 ff., 189 ff., 237 ff. 
nander das vorgelagerte Zulassungsverfahren im Sinne des „Ob“134 sowie das nachgelagerte, an das Wahlrecht angelehnte Abstimmungsprüfungsverfahren zum Zwecke der Verfahrenskontrolle ${ }^{135}$. Zur Inhaltskontrolle können anschließend noch Normenkontrolle und - so landesrechtlich gewährleistet - Verfassungsbeschwerde genutzt werden ${ }^{136}$. Das ist doppelt mißlich: Zunächst illustriert die jüngste Entscheidung des Bayerischen Verfassungsgerichtshofes zum Rauchverbot in sog. Shisha-Cafés ${ }^{137}$, daß die Aufteilung der einzelnen Sachfragen auf Abstimmungsprüfung und Normenkontrolle für den Bürger gelinde gesagt nicht selbsterklärend ist. Wichtiger dürfte sein, daß eine Lücke für den nach meiner Überzeugung künftig häufiger werdenden - Fall be- oder entsteht, daß es während eines laufenden Verfahrens zum Streit kommt (man denke an behördliche Obstruktion ${ }^{138}$ oder eben legislative Ingerenzen nach Thüringer Muster ${ }^{139}$ ). Da Reichweite und Anwendbarkeit der Landes-Organstreitigkeiten hier bestenfalls unklar sind ${ }^{140}$, erscheint eine Generalklausel vorzugswürdig, die nach dem Muster von Hamburg und Sachsen-Anhalt das Landesverfassungsgericht für alle Streitigkeiten über die Durchführung von Volksbegehren und Volksentscheide für zuständig erklärt ${ }^{141}$.

134 Zusammenfassend Rux, Demokratie (Fn. 11), S. 292 ff., 418 ff. - Instruktiv die rechtsvergleichende Perspektive bei G. Biaggini, Eine verzwickte Angelegenheit. Die nachträgliche Überprüfung der Regularität einer eidgenössischen Volksabstimmung, in: Schweizerisches Zentralblatt für Staats- und Verwaltungsrecht 113 (2012), S. 429 ff.

135 Näher W. Gensior, Wahlprüfungs- und ähnliche Beschwerden vor Landesverfassungsgerichten, in: C. Starck/K. Stern (Hrsg.), Landesverfassungsgerichtsbarkeit, Bd. II, 1983, S. 105 (110 f., 113 ff.) sowie Przygode, Rechtsprechung (Fn. 12), S. 170 ff. (zum Volksbegehren), $228 \mathrm{ff}$. (zum Volksentscheid).

136 Hier sollte sich verstehen, daß volksbeschlossene Gesetze in diesen Verfahren keinen anderen Rang haben als die vom Parlament verantworteten Normen: Rux, Demokratie (Fn. 11), S. 332; vgl. auch Przygode, Rechtsprechung (Fn. 12), S. 429 ff., mit umfassender Kritik an jeglichen „Vorrang-Theorien“.

137 BayVerfGH, Entsch. v. 13.9.2011, Az. Vf. 12-VII-10, BayVB1. 2012, 13; auch dokumentiert und kommentiert in: F. Wittreck, Ausgewählte Entscheidungen zur direkten Demokratie, in: Feld u.a., Jahrbuch 2011 (Fn. 15), S. 311.

138 Instruktives Anschauungsmaterial liefern (nicht nur) die Vorwürfe einer unzulässigen Einwirkung auf Eintragung und Abstimmung im Rahmen des Volksbegehrens „,Das bessere Müllkonzept“; vgl. speziell dazu BayVGH, Entsch. v. 28.6.1990, Az. 5 CE 90.1852, BayVB1. 1990, 721; Entsch. v. 13.2.1991, Az. 4 CE 91.404, BayVB1. 1991, 403; BayVerfGH, Entsch. v. 4.2.1991, Az. Vf. 4-IV-91, BayVerfGHE 44, 9 (16 f.); Entsch. v. 19.1.1994, Az. Vf. 89-III-92, Vf. 92-III-92, BayVerfGHE 47, 1 (20, 28 ff.); BVerfGE 96, 231 (234 f.) und dazu Przygode, Rechtsprechung (Fn. 12), S. 179 ff., 183 ff., 450.

139 Vgl. oben bei Fn. 109.

140 Vgl. HambVerfG, Urteil v. 27.4.2007, Az. HVerfG 4/06, LVerfGE 18, 211 (226 ff.).

141 Vgl. Art. 65 Abs. 3 Nr. 5 HambVerf. sowie Art. 75 Nr. 2 SAVerf. und dazu K. David, Verfassung der Freien und Hansestadt Hamburg, 2. Aufl. 2004, Art. 65 Rn. 70 ff. bzw. A. Reich, Verfassung des Landes Sachsen-Anhalt, 2. Aufl. 2004, Art. 75 Rn. 3. 


\section{Schluß: Erfolgt der ,Siegeszug“ der direkten Demokratie nach Plan?}

Wir kommen zum Schluß. Nun soll die Überschrift keineswegs insinuieren, die geschilderte Entwicklung hin zu einer Stärkung der Instrumente der direkten Demokratie sei im hegelschen Sinne vernunftnotwendig ${ }^{142}$. Wer sich wie der Verfasser kategorisch dagegen wendet, etwa bestimmte Prozentsätze für Unterstützungsquoren verbindlich aus der Ewigkeitsklausel abzuleiten ${ }^{143}$, sollte auch nicht den Weltgeist bemühen, wenn die Quoren schließlich doch unter diese angeblich magische Grenze sinken.

Umgekehrt griffe es aber wohl auch zu kurz, den geschilderten Prozeß entweder als zufällig zu etikettieren, ihn bloßen intraföderalen Gesetzesplagiaten zuzuschreiben oder ihn womöglich allein der effektiven Lobbyarbeit des Vereins „Mehr Demokratie“" geschuldet zu sehen ${ }^{144}$.

Vielleicht trifft das sozialwissenschaftliche Konzept der Pfadabhängigkeit es besser ${ }^{145}$ : Augenblicklich zeichnet sich ab, daß die spätestens in Weimar getroffene deutsche Systementscheidung für direkte Demokratie in Form der vollplebiszitären Gesetzgebung mit hoher Wahrscheinlichkeit dazu tendiert, die Hürden direktdemokratischer Instrumente sukzessive zu senken bzw. deren Spielraum Stück für Stück zu erweitern. Und in diesem Sinne spricht viel dafür, daß in einigen Jahren eher Hamburg als das Saarland Vorbild für die direkte Demokratie in der Mehrzahl der Länder sein wird.

142 Die Wendung von der „List der Vernunft“ bei G.W.F. Hegel, Die Vernunft in der Geschichte, herausgegeben von J. Hoffmeister, 5. Aufl. 1955, S. 105; vgl. dazu F. Fulda, Art. List der Vernunft, in: J. Ritter/K. Gründer (Hrsg.), Historisches Wörterbuch der Philosophie, Bd. 5, 1980, Sp. 343.

143 So etwa R. Gröschner, Unterstützungsquoren für Volksbegehren, in: ThürVBl. 2001, S. 193 (197 ff.), der ein landesspezifisches Zehnprozentquorum mit Ewigkeitsrang postuliert.

144 In diese Richtung aber offenbar A. Dänner/R. Huber, Wer wenn nicht wir, in: Mehr Demokratie e.V. (Hrsg.), Das Kuratorium für Mehr Demokratie, 2011, S. 34 ff.

145 Statt aller J. Beyer, Pfadabhängigkeit ist nicht gleich Pfadabhängigkeit!, in: ZfS 34 (2005), S. 5 (5 ff.). 